\title{
Numerical Simulation of Radio Frequency Ablation with State Dependent Material Parameters in Three Space Dimensions
}

\author{
Tim Kröger ${ }^{1}$, Inga Altrogge ${ }^{1}$, Tobias Preusser ${ }^{1}$, Philippe L. Pereira ${ }^{2}$, \\ Diethard Schmidt ${ }^{2}$, Andreas Weihusen ${ }^{3}$, and Heinz-Otto Peitgen ${ }^{1,3}$ \\ ${ }^{1} \mathrm{CeV}$ is - Center for Complex Systems and Visualization, \\ University of Bremen, Germany \\ \{kroeger, altrogge, preusser, peitgen\}@cevis.uni-bremen.de \\ ${ }^{2}$ Dept. of Diagnostic Radiology, Eberhard Karls University, Tübingen, Germany \\ \{philippe.pereira, diethard.schmidt\}@med.uni-tuebingen.de \\ $3 \mathrm{MeV}$ is - Center for Medical Diagnostic Systems and Visualization, \\ Bremen, Germany \\ \{weihusen, peitgen\}@mevis.de
}

\begin{abstract}
We present a model for the numerical simulation of radio frequency (RF) ablation of tumors with mono- or bipolar probes. This model includes the electrostatic equation and a variant of the well-known bio-heat transfer equation for the distribution of the electric potential and the induced heat. The equations are nonlinearly coupled by material parameters that change with temperature, dehydration and damage of the tissue. A fixed point iteration scheme for the nonlinear model and the spatial discretization with finite elements are presented. Moreover, we incorporate the effect of evaporation of water from the cells at high temperatures using a predictor-corrector like approach. The comparison of the approach to a real ablation concludes the paper.
\end{abstract}

\section{Introduction}

Minimally invasive treatment of tumors has become a promising alternative to other therapy forms. In particular for cases where surgical resection is too complex or even impossible, thermo-therapies such as radio frequency ( $\mathrm{RF}$ ) ablation are applied: One or more probes are placed in the tumor, producing an electric current that warms the neighboring tissue. When a critical temperature $\left(\approx 54^{\circ} \mathrm{C}\right)$ is exceeded, proteins denaturate and the tissue is destroyed. To increase the coagulation volume, much higher temperatures are generated near the probes. This leads to evaporation of water in the cells (as can be seen through ultrasound monitoring of the ablation process) and to dehydration of the tissue.

For the planning of RF ablation, the simulation of this treatment is an indispensable tool. The results of such simulations can provide information about a suitable probe position, power setup, and ablation time. The simulation of RF ablation (and related thermo-therapies) has been considered by several authors [1 \begin{tabular}{ll|l|l|l}
1 & 2 & 3 & 4 & 5
\end{tabular} . Many approaches assume constant material parameters, exclude evaporation of water 23 , and use commercial grid generators and finite element software for the discretization of the resulting linear equations. Stein [1] 
presented an approach which takes into account non-constant material parameters and evaporation but assumes a cylindrical symmetry around the probe. His discretization uses finite differences and explicit time-stepping. Several authors investigate the effect and the modeling of blood flow for RF ablation [367].

The results presented in this paper are part of a collaboration of $\mathrm{CeV}$ is and $\mathrm{MeV}$ is and a variety of clinical partners working together in publicly funded research project:1. The main goal of these projects are the development and the evaluation of clinically useful computer assisted systems for planning, monitoring, and assessing of tumor ablation. A complete destruction of tumor tissue is essential for treatment success, similar to $\mathrm{R} 0$ resection in liver tumor surgery. Here, the numerical simulation of RF ablation for procedure planning can considerably contribute to a successful ablation. At CeVis, a group headed by the third author is developing advanced numerical simulation procedures.

Our current model of RF ablation combines features of several existing models: It is a three-dimensional, nonlinear finite element model incorporating material parameters changing with temperature, dehydration, and damage. For the evaporation, we consider a simple two-phase model. This enables us to model the nonlinear behavior of the electric generator as a consequence of the dehydration (an effect that is known to change the ablation process essentially). Patientindividual segmented vascular systems are considered as well. Moreover, the discretization allows to simulate several simultaneously applied mono- or bipolar RF probes in any geometric constellation; a novelty is the usage of Robin boundary conditions for the electric potential at the domain boundary.

\section{A Mathematical Model of Radio Frequency Ablation}

Let us begin with a description of the geometrical setting: We consider a cuboidshaped domain $\Omega \subset \mathbb{R}^{3}$ with (outer) boundary $\Gamma_{\text {out }}=\partial \Omega$. We assume (cf. Fig.1) that one or more probes are applied within $\Omega$, the union of which will be denoted by $\Omega_{\mathrm{pr}}$. The probes consist of insulating material and electrodes which are either positively or negatively charged. We let $\Omega_{+}, \Omega_{-} \subset \Omega_{\mathrm{pr}}$ denote the union of all positive and negative electrodes, respectively, and $\Omega_{ \pm}=\Omega_{+} \cup \Omega_{-}$.

The mathematical model for the simulation of RF ablation consists of three interacting parts: The first part models the energy applied to the tissue by the probe and the electric generator. The second part considers the heat distribution in the tissue including sources and sinks and the phase change. The third part tackles the effect of the heat on the (malignant) cells and their destruction. These parts of the ablation model depend on a variety of material parameters, which themselves depend on the various states of the tissue.

Thus, denoting the time-space computational domain by $W=I \times \bar{\Omega}$ where $I=\left[0, t_{\max }\right], t_{\max }>0$, and by $C(D, E)$ the space of continuous functions $D \rightarrow$ $E$, we are looking for the following five states:

${ }^{1}$ LITT/RFITT funded by the German Research Foundation (Pe 199/11-1 and Pe 199/15-2) and the national research networks VICORA and FUSION funded by the Federal Ministry of Education and Research (01EZ0401 and 01IBE03C). 
- electric potential $\varphi \in C(W, \mathbb{R})$ of the probe induced by the generator,

- temperature distribution $T \in C(W, \mathbb{R})$,

- relative content of fluid water $F_{\mathrm{W}} \in C(W,[0,1])$,

- relative content of vapor $F_{\mathrm{V}} \in C(W,[0,1])$,

- coagulation state $F_{\mathrm{C}} \in C(W,[0,1])$,

and the following four material parameters:

- electric conductivity $\sigma \in C(W, \mathbb{R})$, • density $\rho \in C(W, \mathbb{R})$,

- specific heat capacity $c \in C(W, \mathbb{R})$, - thermal conductivity $\lambda \in C(W, \mathbb{R})$.

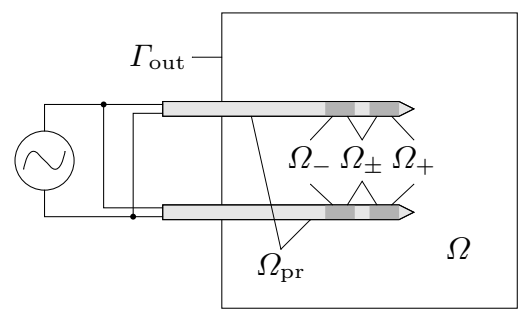

Fig. 1. Schematic geometrical setting. Note that $\Omega_{+} \cup \Omega_{-}=\Omega_{ \pm} \subset \Omega_{\mathrm{pr}} \subset \Omega$.

Let us give some remarks on the state variables $F_{\mathrm{W}}$ and $F_{\mathrm{V}}$ : The evaporation of water is an important effect which leads to a drying-up of the tissue and consequently to a reduced conductivity for the electric current. The quantities $F_{\mathrm{W}}$ and $F_{\mathrm{V}}$ specify how much of the cell-volume available for water is actually filled by fluid water or vapor. When water evaporates, we assume that the surplus vapor will pass off due to the pressure and condensate in remote regions. On the other hand, if condensation takes place (e.g., if electrodes are switched off), the tissue will contract temporarily and water will only slowly rediffuse to fill the free space. Hence, instead of the intuitive relation $F_{\mathrm{W}}+F_{\mathrm{V}}=1$, we only assume

$$
F_{\mathrm{W}}(t, x)+F_{\mathrm{V}}(t, x) \leq 1 \quad \text { for all }(t, x) \in W .
$$

Material Parameters. Experimental investigations [1] have shown that all of the material parameters (2) depend nonlinearly on $T, F_{\mathrm{W}}$, and $F_{\mathrm{C}}$. But this dependence is not known in every detail and moreover it may vary for each patient individually. However, Stein [1] has shown that model equations can be used that are linear in each of $T, F_{\mathrm{W}}$, and $F_{\mathrm{C}}$, i. e.

$$
\sigma(t, x)=\sigma_{1} \cdot\left(1+\sigma_{2}\left(T(t, x)-T_{\text {body }}\right)\right)\left(1+\sigma_{3}\left(1-F_{\mathrm{W}}(t, x)\right)\right)\left(1+\sigma_{4} F_{\mathrm{C}}(t, x)\right)
$$

and analogously for $\rho, c$, and $\lambda$. Note that we abuse notation and write $\sigma(t, x)$ instead of $\sigma\left(T(t, x), F_{\mathrm{W}}(t, x), F_{\mathrm{C}}(t, x)\right)$. Following Stein, we use the constants

$$
\begin{aligned}
& \sigma_{1}=0.21 \mathrm{~A} / \mathrm{Vm}, \quad \sigma_{2}=0.013 \mathrm{~K}^{-1}, \quad \sigma_{3}=-1, \quad \sigma_{4}=1.143, \\
& \rho_{1}=1080 \mathrm{~kg} / \mathrm{m}^{3}, \quad \rho_{2}=-0.00056 \mathrm{~K}^{-1}, \quad \rho_{3}=-0.657, \quad \rho_{4}=0, \\
& c_{1}=3455 \mathrm{~J} / \mathrm{gK}, \quad c_{2}=0, \quad c_{3}=-0.376, \quad c_{4}=0, \\
& \lambda_{1}=0.437 \mathrm{~W} / \mathrm{Km}, \quad \lambda_{2}=0.0025 \mathrm{~K}^{-1}, \quad \lambda_{3}=0, \quad \lambda_{4}=0
\end{aligned}
$$


( $\sigma_{3}=-1$ models the effect of drying-up on the conductivity: $\sigma \rightarrow 0$ as $F_{\mathrm{W}} \rightarrow 0$ ).

Electric Energy. The energy applied by the probe is given by the time integral of the power $p(t, x)=\sigma(t, x)|\nabla \varphi(t, x)|^{2}$. For the frequencies used in RF-ablation, the electric potential $\varphi$ is quasistatic [1] and modeled by

$$
\begin{aligned}
-\nabla \cdot(\sigma \nabla \varphi) & =0 & & \text { in } I \times\left(\Omega \backslash \overline{\Omega_{ \pm}}\right), \\
\varphi & = \pm 1 & & \text { on } I \times \overline{\Omega_{ \pm}} \text {(fixed potential on electrodes), } \\
n \cdot \nabla \varphi & =\frac{n \cdot(s-x)}{|s-x|^{2}} \varphi & & \text { on } I \times \Gamma_{\text {out }} \text { (Robin boundary conditions). }
\end{aligned}
$$

Condition (4c) (where $n$ denotes the outer normal to $\Gamma_{\text {out }}$ ) enables us to model bipolar as well as monopolar probes without prescribing a neutral potential on the boundary $\Gamma_{\text {out }}$ as e.g. in [3]. The condition corresponds to the assumption that on $\Gamma_{\text {out }}$ (i.e. far away from the probe), the potential behaves as it was induced by a point load at the barycenter $s$ of the union of all probes.

The values \pm 1 on $\overline{\Omega_{ \pm}}$are chosen arbitrarily. The correct potential induced by the generator is obtained by a scaling, which also takes into account the nonlinear behavior of the generator due to the tissue resistance $R_{\mathrm{tis}}(t)$. Denoting the setup power of the generator with $p_{\text {setup }}$ (typically in the range $20-200 \mathrm{~W}$ ), we have

$$
p_{\text {total }}(t)=\int_{\Omega} p(t, x) d x \quad, \quad p_{\text {eff }}(t)=\frac{4 p_{\text {setup }} R_{\mathrm{tis}}(t) R_{\mathrm{I}}}{\left(R_{\mathrm{tis}}(t)+R_{\mathrm{I}}\right)^{2}}, \quad R_{\mathrm{tis}}(t)=\frac{U^{2}}{p_{\text {total }}(t)},
$$

where $R_{\mathrm{I}}$ is the inner resistance of the generator and $U$ is the difference of the potential $\varphi$ on the two electrodes $(U=2 \mathrm{~V}$ for bipolar and $U=1 \mathrm{~V}$ for monopolar probes). From these quantities, we compute $Q_{\mathrm{rf}}(t, x)=p(t, x) \cdot p_{\text {eff }}(t) / p_{\text {total }}(t)$.

Temperature. The model equation for the temperature distribution is

$$
\begin{aligned}
\partial_{t}(\rho c T)-\nabla \cdot(\lambda \nabla T) & =Q & & \text { in } I \times\left(\Omega \backslash \overline{\Omega_{\mathrm{pr}}}\right), \\
T & =T_{\text {body }} & & \text { on } I \times \overline{\Omega_{\mathrm{pr}}}(\text { cooled probe }), \\
n \cdot \nabla T & =0 & & \text { on } I \times \Gamma_{\text {out }} \text { (natural boundary conditions) },
\end{aligned}
$$

which is known as heat transfer equation, with the initial value $T(t=0) \equiv$ $T_{\text {body }}=37^{\circ} \mathrm{C}$. The right hand side is $Q(t, x)=Q_{\mathrm{rf}}(t, x)+Q_{\text {perf }}(t, x)+Q_{\mathrm{pc}}(t, x)$, where $Q_{\text {rf }}$ has been introduced above.

$Q_{\text {perf }}$ models the cooling effect due to perfusion. Various authors have studied the modeling of perfusion in the context of RF ablation 6 6]. In our approach, we consider the capillary blood flow having diffusive effects on the temperature only. Thus, we use an extended version of Pennes' approach [9] and set $Q_{\text {perf }}(t, x)=\nu(x) \rho_{\text {blood }} c_{\text {blood }}\left(T_{\text {body }}-T(t, x)\right)$ if $F_{\mathrm{C}}(t, x)<F_{C}^{*}:=1-\exp (-1)$ and $Q_{\text {perf }}(t, x)=0$ else. However, we use a spatially varying perfusion coefficient $\nu(x)$ which is $0.05 \mathrm{~s}^{-1}$ inside the hepatic and the portal vein, $0.1 \mathrm{~s}^{-1}$ inside the artery, and $0.01765 \mathrm{~s}^{-1}$ else [1].

$Q_{\mathrm{pc}}$ is the heat source/sink due to the phase change of water. Modeling the thermodynamics of a phase change would involve further differential equations 
for the pressure and thus increase the computational complexity. Instead, we use a predictor-corrector like approach which is described in the next section.

Coagulation. The denaturation of tissue due to coagulation is modeled following the Arrhenius formalism (cf. [101]). This leads to

$$
F_{\mathrm{C}}=1-\exp (-W), \quad \partial_{t} W=A_{\mathrm{A}} \exp \left(-E_{\mathrm{A}}(R T)^{-1}\right), \quad W\left(t_{0}\right)=0,
$$

where $R$ is the universal gas constant and $A_{\mathrm{A}}=9.4 \cdot 10^{104} \mathrm{~s}^{-1}$ and $E_{\mathrm{A}}=6.7$. $10^{5} \mathrm{~J} / \mathrm{mol}$ are the corresponding Arrhenius parameters.

\section{Discretization}

After having introduced our model, we now explain how the differential equations are discretized. This section consists of three parts: We first present an overview of our overall discretization scheme in space and time and supply the details in the second part. The last part deals with the discrete phase change model.

For the spatial discretization we work with the weak forms of (44) and (5):

$$
\begin{array}{ll}
0=\int_{\Omega \backslash \overline{\Omega_{ \pm}}} \sigma \nabla \varphi \cdot \nabla v d x-\int_{\Gamma_{\mathrm{out}}} \frac{n \cdot(s-x)}{|s-x|^{2}} \sigma \varphi v d s(x) & \forall v \in H_{ \pm}^{1}:=H_{\overline{\Omega_{ \pm}}}^{1}, \\
0=\int_{\Omega \backslash \overline{\Omega_{\mathrm{pr}}}}\left(\partial_{t}(\rho c T) v+\lambda \nabla T \cdot \nabla v-Q v\right) d x & \forall v \in H_{\mathrm{pr}}^{1}:=H_{\overline{\Omega_{\mathrm{pr}}}}^{1},
\end{array}
$$

Dividing $\Omega$ uniformly into a finite number of cuboids we introduce the finite element space $\mathcal{V}^{h}$ of globally continuous, cell-wise trilinear functions.

Next, we introduce our overall time discretization. Let $\mathcal{X}=\left(\mathcal{V}^{h}\right)^{5}$ denote the state space (i. e. any $x \in \mathcal{X}$ consists of all five states (1) in their respective finite-dimensional function space at a particular time level). For the solution of our nonlinear system, we have to deal with an $\mathcal{X}$-valued initial value problem:

Find $x: I \rightarrow \mathcal{X}$ such that $\partial_{t} x=\mathcal{F}(x)$ and $x\left(t_{0}\right)=x_{0}$.

We approximate the solution by a sequence of discrete time levels $x^{n} \approx x\left(t_{n}\right)$, $t_{n}=t_{n-1}+\tau_{n}$ computed by the implicit Euler scheme $x^{n}=x^{n-1}+\tau_{n} \mathcal{F}\left(x^{n}\right)$. This equation is solved using a fixed point iteration

$$
x^{n, k+1}=x^{n, k}+\tau_{n} \mathcal{G}\left(x^{n-1}, x^{n, k}, \tau_{n}\right), \quad x^{n, 0}=x^{n-1},
$$

where $\mathcal{G}\left(x^{n-1}, x^{n, k}, \tau_{n}\right)$ is some semi-implicit approximation to $\mathcal{F}\left(x^{n}\right)$ to be explained below. When $\left\|x^{n, k}-x^{n, k-1}\right\|$ is below a given threshold for some $k$, then we set $x^{n}=x^{n, k}$ for this $k$. If it fails to do so up to some given $k_{\max }$, we multiply $\tau_{n}$ with $\frac{1}{2}$ and restart (9). This is repeated until convergence is achieved.

Fixed Point Iteration. We will now define our fixed point iteration function $\mathcal{G}$ by explaining how $\left(\varphi, T, F_{\mathrm{W}}, F_{\mathrm{V}}, F_{\mathrm{C}}\right)^{n, k+1}$ is computed. We use a semi-implicit approach, i. e. $\varphi^{n, k+1}$ and $T^{n, k+1}$ are chosen such that 


$$
\begin{aligned}
& 0=\int_{\Omega \backslash \overline{\Omega_{ \pm}}} \sigma^{n, k} \nabla \varphi^{n, k+1} \cdot \nabla v d x-\int_{\Gamma_{\mathrm{out}}} \frac{n \cdot(s-x)}{|s-x|^{2}} \sigma^{n, k} \varphi^{n, k+1} v d s(x), \\
& 0=\int_{\Omega \backslash \overline{\Omega_{\mathrm{pr}}}}\left(\frac{\rho^{n, k} c^{n, k}}{\tau_{n}}\left(T^{n, k+1}-T^{n-1}\right) v+\lambda^{n, k} \nabla T^{n, k+1} \cdot \nabla v-Q^{n, k} v\right) d x
\end{aligned}
$$

for all $v \in \mathcal{V}_{ \pm}^{h}$ and $v \in \mathcal{V}_{\mathrm{pr}}^{h}$, respectively. Equations (10) and (11) each lead to a system of linear equations, which are solved using a conjugate gradient solver. To complete the formula for $T^{n, k+1}$, we need to state our discretization of $Q^{n, k}$.

For the discretization of $Q_{\mathrm{rf}}^{n, k}$, we note that $\sigma$ and $\varphi$ are approximated by $\sigma^{n, k}$ and $\varphi^{n, k+1}$, and the $\nabla$ operator is discretized by the monotonized centered slope limiter [11]. For $Q_{\text {perf }}^{n, k}$, we note that $F_{\mathrm{C}}$ and $T$ are approximated by $F_{\mathrm{C}}^{n, k}$ and $T^{n, k+1}$, which changes the matrix of the linear system (11). Finally, the discretization of $F_{\mathrm{C}}^{n, k+1}$ is done in a straightforward manner.

Phase Change. Note that $F_{\mathrm{W}} \rightarrow 0$ implies $Q_{\mathrm{rf}} \rightarrow 0$, i. e. there will not be any heat source any more, and hence, the temperature is always bounded by $T_{\text {boil }}$.

It is important to let the temperature increase up to $T_{\text {boil }}$ without evaporation (i. e. $Q_{\mathrm{pc}}=0$ ). If this point has been reached, the temperature has to be fixed as long as there is still a heat source or $F_{\mathrm{V}}(t, x)>0$. We perform the whole phase change procedure as a post-processing step: We first solve a modified heat equation in which $Q_{\mathrm{pc}}$ is omitted, denoting the result as $\tilde{T}^{n, k+1}$, and then compute $Q_{\mathrm{pc}}^{n, k}$ such that $T(t, x)$ is fixed at $T_{\text {boil }}$ whenever this is necessary:

$$
Q_{\mathrm{pc}}^{n, k}(t, x)=\frac{\rho^{n, k}(t, x) c^{n, k}(t, x)}{\tau_{n}} \min \left(T_{\mathrm{boil}}-\tilde{T}^{n, k+1}(t, x), \frac{F_{\mathrm{V}}^{n, k}(t, x) E_{\mathrm{vap}} q_{\mathrm{vap}}}{c^{n, k}(t, x)}\right) .
$$

Then, we set $T^{n, k+1}(t, x)=\tilde{T}^{n, k+1}(t, x)+\tau_{n} Q_{\mathrm{pc}}^{n, k}(t, x) /\left(\rho^{n, k}(t, x) c^{n, k}(t, x)\right)$ and

$$
\begin{aligned}
& \tilde{F}_{\mathrm{W}}^{n, k+1}(t, x)=\max \left(F_{\mathrm{W}}^{n-1}(t, x)+\tau_{n} \frac{Q_{\mathrm{pc}}^{n, k}(t, x)}{E_{\mathrm{vap}} \rho^{n, k}(t, x)}, 0\right), \\
& F_{\mathrm{V}}^{n, k+1}(t, x)=\min \left(F_{\mathrm{V}}^{n-1}(t, x)-\tau_{n} \frac{Q_{\mathrm{pc}}^{n, k}(t, x)}{E_{\mathrm{vap}} q_{\mathrm{vap}} \rho^{n, k}(t, x)}, 1-\tilde{F}_{\mathrm{W}}^{n, k+1}(t, x)\right), \\
& F_{\mathrm{W}}^{n, k+1}(t, x)=\frac{\tau_{A} \tilde{F}_{\mathrm{W}}^{n, k+1}(t, x)+\tau_{n}\left(1-F_{\mathrm{V}}^{n, k+1}(t, x)\right)}{\tau_{A}+\tau_{n}} .
\end{aligned}
$$

Equations (12) and (13) model the change of the water and vapor content which is due to the phase change; here, $E_{\text {vap }}=2.256 \cdot 10^{6} \mathrm{~J} / \mathrm{kg}$ is the specific enthalpy of evaporation and $q_{\mathrm{vap}}=1 / 332$ is the density ratio of vapor and water. The second term in (13) asserts (3). Finally, (14) describes an exponential flow-back (with speed determined by $\tau_{A}=4 \mathrm{~s}$ ) of water after the condensation process [1].

\section{Numerical Results}

For an initial evaluation of our algorithm, we compared the results to a data set from the University Hospital of Tübingen. Vascular system and tumor were 

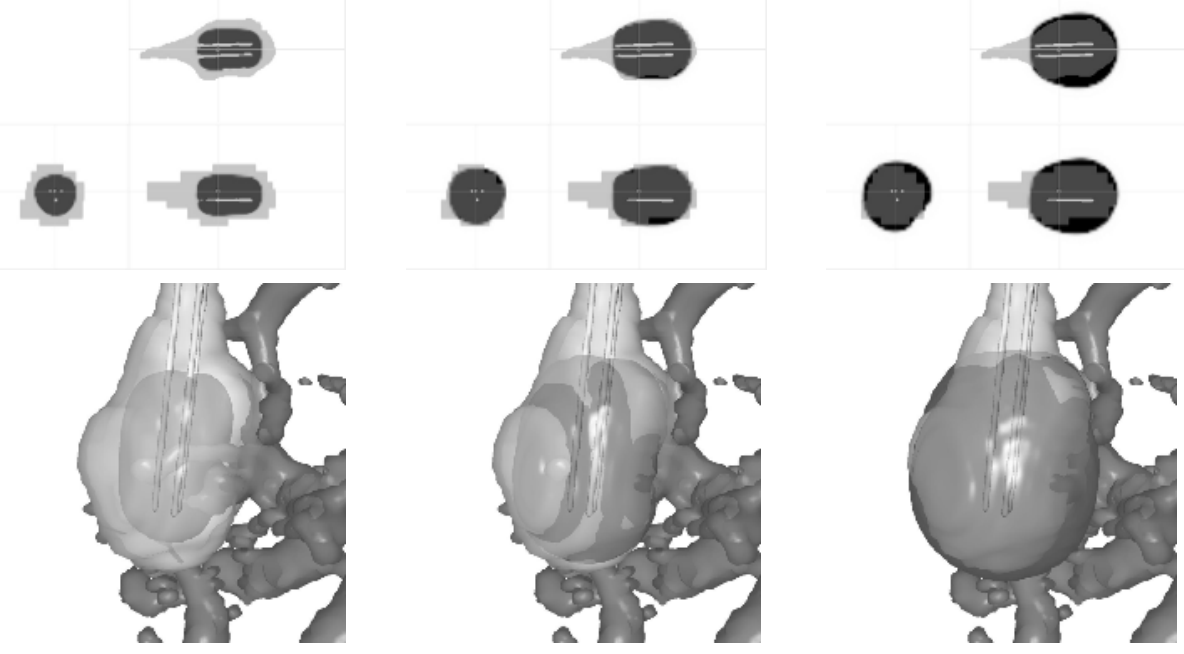

Fig. 2. Top: Overlay of the computed coagulation after 2, 5, and 8 minutes (dark) and the segmented coagulation (light). Bottom: Three-dimensional view including segmented vessel system and probes. (Images can be found at ww.mevis.de/ tim/)

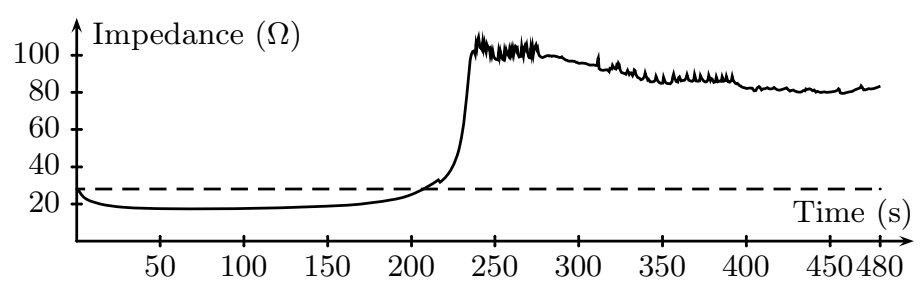

Fig. 3. Computed tissue impedance during the ablation

segmented from a pre-interventional CT scan [12]; ablation parameters were set up as recorded; and the coagulation was compared with a post-interventional CT scan, which, however, had been performed two months after the intervention.

Ablation parameters were a "Cool-Tip ${ }^{\mathrm{TM}}$ M RF CTCM-1525" cluster (three monopolar RF applicators in a fixed geometrical arrangement), a Radionics generator (set to its maximum power $p_{\text {setup }}=200 \mathrm{~W}$ at a frequency of $480 \mathrm{kHz}$ and an inner resistance of $R_{\mathrm{I}}=80 \Omega$ ), and 8 minutes ablation time. Our computational domain of approx. $60 \times 40 \times 40 \mathrm{~mm}$ was covered by interleaved grids of grid sizes of about $0.2 \mathrm{~mm}, 0.4 \mathrm{~mm}$, and $0.8 \mathrm{~mm}$. We chose a time step size of $1 \mathrm{~s}$, that was reduced to $0.5 \mathrm{~s}$ (by the implicit time stepping scheme with $k_{\max }=5$ ) in 231 cases and to $0.25 \mathrm{~s}$ in 2 cases. The computation took 6 hours.

Figure 2 shows simulation results. Since the post-interventional CT scan had been made two months later, the liver had already begun to regenerate, which might explain why the segmented lesion is slightly too small. Note also that the 
'handle' in the segmented lesion was produced by leaving the generator switched on while pulling out the probes; a process not included in the simulation.

Figure 3 shows the computed tissue impedance progression. After about $230 \mathrm{~s}$, the impedance increases drastically, because a layer of dried-up tissue has been formed around the probes. For models that do not incorporate evaporation of water, the impedance would stay constant (dashed line), a non-physical behavior.

\section{Discussion}

We presented a mathematical model of RF ablation and its discretization, combining the advantages of several different approaches. Also, to our knowledge, the proposed handling of monopolar probes and our evaporation model are new. In a preliminary application to patient data, the scheme yielded reasonable results. Future work will cover a more systematic evaluation as well as extensions such as error estimation, unstructured grids, and blood advection.

The authors thank the VICORA team and in particular T. Stein and A. Roggan from Celon AG for valuable hints and fruitful discussions on the topic. Also, we would like to thank the team from MeVis, especially S. Zentis and C. Hilck for preprocessing the CT scans.

\section{References}

1. Stein, T.: Untersuchungen zur Dosimetrie der hochfrequenzstrominduzierten interstitiellen Thermotherapie in bipolarer Technik. Volume 22 of Fortschritte in der Lasermedizin. Müller and Berlien (2000)

2. Tungjitkusolum, S., Staelin, S.T., Haemmerich, D., et al.: Three-dimensional finiteelement analyses for radio-frequency hepatic tumor ablation. IEEE Trans. Biomed. Eng. 49 (2002) 3-9

3. Welp, C., Werner, J.: Large vessel cooling in hepatic radiofrequency ablation: investigation on the influence of blood flow rate. (IEEE Trans. Biomed. Eng.) submitted.

4. Deuflhard, P., Weiser, M., Seebaß, M.: A new nonlinear elliptic multilevel fem applied to regional hyperthermia. Comput. Vis. Sci. 3 (2000) 115-120

5. Roggan, A.: Dosimetrie thermischer Laseranwendungen in der Medizin. Volume 16 of Fortschritte in der Lasermedizin. Müller and Berlien (1997)

6. Jain, M.K., Wolf, P.D.: A three-dimensional finite element model of radiofrequency ablation with blood flow and its experimental validation. Ann. Biomed. Eng. 28 (2000) 1075-84

7. Preusser, T., Weihusen, A., Peitgen, H.O.: On the modelling of perfusion in the simulation of RF-ablation. In: Proceedings of Simulation and Visualization (SimVis), Magdeburg (2005)

8. Jain, R. K., Grantham, F. H., Gullion, P. M.: Blood flow and heat transfer in walker 256 mammary carcinoma. J. Natl. Cancer Inst. 69 (1979) 927-931

9. Pennes, H.H.: Analysis of tissue and arterial blood temperatures in a resting forearm. J. Appl. Physiol. 1 (1948) 93-122 
10. Arrhenius, S.: Über die Reaktionsgeschwindigkeit bei der Inversion von Rohrzucker durch Säuren. Z. Phys. Chem 4 (1889) 226-248

11. LeVeque, R.J.: Wave propagation algorithms for multidimensional hyperbolic systems. J. Comp. Phys. 131 (1997) 327-353

12. Schenk, A., Prause, G., Peitgen, H.O.: Efficient semiautomatic segmentation of 3D objects in medical images. In: Proc. MICCAI. Volume 1935 of Springer LNCS. (2000) 186-195 